\title{
Hydrogen Retention in Lithium on Metallic Walls from "In Vacuo" Analysis in LTX and Implications for High-Z Plasma-Facing Components in NSTX-U
}

\author{
R. Kaita, ${ }^{1}$ M. Lucia, ${ }^{1}$ J. P. Allain, ${ }^{2}$ F. Bedoya,${ }^{2}$ R. Bell,${ }^{1}$ D. Boyle,${ }^{1}$ \\ A. Capece, ${ }^{3}$ M. Jaworski, ${ }^{1}$ B. E. Koel,${ }^{4}$ R. Majeski, ${ }^{1}$ J. Roszell, ${ }^{4}$ \\ J. Schmitt, ${ }^{1}$ F. Scotti, ${ }^{5}$ C. H. Skinner, ${ }^{1}$ V. Soukhanovskii ${ }^{5}$
}

\begin{abstract}
The application of lithium to plasma-facing components (PFCs) has long been used as a technique for wall conditioning in magnetic confinement devices to improve plasma performance. Determining the characteristics of PFCs at the time of exposure to the plasma, however, is difficult because they can only be analyzed after venting the vacuum vessel and removing them at the end of an operational period. The Materials Analysis and Particle Probe (MAPP) addresses this problem by enabling PFC samples to be exposed to plasmas, and then withdrawn into an analysis chamber without breaking vacuum. The MAPP system was used to introduce samples that matched the metallic PFCs of the Lithium Tokamak Experiment (LTX). Lithium that was subsequently evaporated onto the walls also covered the MAPP samples, which were then subject to LTX discharges. In vacuo extraction and analysis of the samples indicated that lithium oxide formed on the PFCs, but improved plasma performance persisted in LTX. The reduced recycling this suggests is consistent with separate surface science experiments that demonstrated deuterium retention in the presence of lithium oxide films. Since oxygen decreases the thermal stability of the deuterium in the film, the release of deuterium was observed below the lithium deuteride dissociation temperature. This may explain what occurred when lithium was applied to the surface of the NSTX Liquid Lithium Divertor (LLD). The LLD had segments with individual heaters, and the deuterium-alpha emission was clearly lower in the cooler regions. The plan for NSTX-U is to replace the graphite tiles with high-Z PFCs, and apply lithium to their surfaces with lithium evaporation. Experiments with lithium coatings on such PFCs suggest that deuterium could still be retained if lithium compounds form, but limiting their surface temperatures may be necessary.
\end{abstract}

Keywords: lithium, plasma-surface interactions, materials science and technology

\section{Introduction}

The value of lithium plasma-facing components (PFC) to improve plasma performance has long been appreciated. For example, lithium wall conditioning produced the largest increase in the fusion "triple product," $i$. e., the product of density, confinement time, and temperature, in the Tokamak Fusion Test Reactor (TFTR).[1] Approaches for introducing lithium into TFTR included lithium pellet injection and laser ablation of lithium in an in-vessel crucible. The locations that served as limiters for TFTR discharges depended on the plasma radius. When the radius was varied, different PFC surfaces could be coated with lithium from the plasma in a process called "painting." The PFCs in TFTR were made of graphite, including the surfaces that served as plasma limiters. Over a decade later, lithium was evaporated onto the graphite PFCs of the National Spherical Torus Experiment (NSTX). Unlike TFTR, NSTX operated with divertor plasmas, and improvements to discharge performance were observed as well.[2] 
Experiments with lithium walls have also been conducted in tokamaks with metal ("high-Z") walls. The PFCs in the Current Drive Experiment-Upgrade (CDX-U) were stainless steel, and a fully-toroidal "tray" at the bottom of the vacuum vessel was filled with liquid lithium to serve as a limiter. Lithium evaporated from the tray also coated up to $50 \%$ of the plasma-contacting area. When this occurred, the largest increase over predictions for energy confinement scaling in Ohmic tokamak plasmas was achieved.[3] The CDX-U device was modified to include a conducting shell that conforms to the plasma shape and renamed the Lithium Tokamak Experiment (LTX). The shell has a stainless steel liner that forms the LTX PFC. As in CDX-U, discharge performance was enhanced with the application of lithium wall coatings.[4] The relationship between plasma performance and recycling was inferred from variations in the hydrogen-alpha emission as PFC conditions changed.

Open questions remain, however, about the relationship between the detailed characteristics of the PFCs themselves and plasma performance. An example of why they are difficult to answer is presented in Section 2, where the complexities of the deuterium retention mechanism when lithium is applied to graphite PFCs are described. As a more expeditious means for determining PFC characteristics that better reflect conditions during plasma operations, a diagnostic called the Materials Analysis and Particle Probe (MAPP) was developed. A description of MAPP and its use in characterizing LTX PFCs with lithium coatings is provided in Section 3. The results of surface analysis of samples exposed to lithium evaporation in LTX are also included. Conclusions and implications for lithium coatings on high-Z PFCs are presented in Section 4.

\section{Challenges of Characterizing Lithium Applied to Plasma Facing Components}

Explanations for the improved confinement with the application of lithium to PFCs in magnetic confinement devices appeared to be straightforward. Lithium is a highly reactive metal that is expected to readily bind hydrogenic species. This leads to low-recycling walls, which in turn results in hotter edge plasmas and broader electron temperature profiles. Electron temperature gradients (ETGs) have long been associated with drift wave instabilities that could degrade energy confinement [5]. Reducing wall recycling would reduce ETGs, and thus improve plasma performance.

Low recycling walls were also needed for so-called "supershots" in TFTR, and lithium pellet injection was a technique for achieving them.[6] The favorable confinement scalings of the supershots have been explained through the reduction of turbulence through sheared flows. The reduced ion thermal diffusion from suppression of the toroidal ion temperature gradient (ITG) driven modes then led to the high ion temperatures obtained in supershots.[7]

The choice of lithium as a PFC then seemed to be clear. The high chemical reactivity of lithium enabled retention of hydrogen from the plasma by the formation of lithium hydride. This simple picture should not hold, however, if the lithium were applied to graphite PFCs. Lithium intercalates into graphite, and does not have the same characteristics as lithium in its elemental form. As a result, lithium intercalated into the graphite should not necessarily volumetrically convert to lithium deuteride as observed when a macroscopically-thick $(5 \mathrm{~mm})$ liquid lithium sample was exposed to a high deuterium fluence.[8]

The actual mechanism for the retention of deuterium in graphite PFCs was determined only after analysis of NSTX PFCs. X-ray photoelectron spectroscopy (XPS) measurements were performed after the removal of lithium compounds formed after exposure to air. They revealed chemical complexes involving lithium, oxygen, and carbon.[9] Quantum-classical molecular dynamics (QCMD) modeling was conducted for deuterium bombardment of surface matrices of mixed atoms. Simulations were performed for surfaces composed of lithium, oxygen, and carbon as determined from XPS data. Calculations of deuterium bombardment were also conducted for surfaces consisting only of lithium and carbon.[10]

When lithium and carbon alone were included in the QCMD calculations, the percentage of bound deuterium that shared charges with lithium was even lower than the 
surface fraction of lithium relative to the carbon. This is consistent with what would be expected when lithium intercalates into graphite and binds with carbon. For a surface that included lithium, oxygen, and carbon, however, the percentage of deuterium that shared charges with lithium was again low. The fraction that shared charges with oxygen, however, was not only greater than the percentage of deuterium, but exceeded the fraction of oxygen in the original surface composition.

The QCMD results were thus able to account for the unexpected effectiveness of lithium coatings on graphite to retain deuterium. They demonstrated that binding deuterium in a system that includes lithium, carbon, and oxygen was much more effective for deuterium retention than lithium deuteride formation. The data that supported the modeling, however, were only obtained after the end of an entire NSTX operational period. A PFC tile had to be taken out of the vacuum vessel and treated to remove the lithium compounds that formed after prolonged exposure to air. It then had to be assumed that the exposed surface reflected PFC conditions during plasma operations. This demonstrates the value of analyzing PFC samples immediately after plasma exposure, to reduce the uncertainties from assumptions needed to interpret surfaces exposed to an entire run.

\section{Hydrogen Retention by Lithium Oxide from In Vacuo Sample Exposure and Analysis with Materials Analysis and Particle Probe}

To address the need for in vacuo PFC analysis, i. e., without venting the vacuum vessel and removing PFCs, the MAPP was developed.[11] This system was designed to permit up to four samples to be exposed to plasmas. The samples can then be withdrawn, without breaking vacuum, into an analysis chamber equipped with several surface analysis capabilities. They include $\mathrm{x}$-ray photoelectron spectroscopy (XPS), low-energy ion scattering spectroscopy (LEISS), and direct recoil spectroscopy (DRS). Because the samples are attached to heaters that can be controlled separately, they can be analyzed individually with thermal desorption spectroscopy (TDS) or temperature programmed desorption (TPD).
The MAPP diagnostic was originally constructed for use on NSTX. During the outage for the construction of the NSTX-Upgrade (NSTX-U), the MAPP was installed on LTX to study samples introduced into the plasma chamber.[12] The samples were made of materials that included stainless steel to match the conducting shell surface that constitute the LTX PFCs. Lithium evaporated onto LTX walls also covered the samples. These samples were subsequently exposed to LTX discharges, and withdrawn into analysis chamber under vacuum and analyzed using XPS.

Figure 1a shows the atomic ratios of lithium to oxygen $\left(\mathrm{R}_{\mathrm{Li}, \mathrm{O}}\right)$, as determined from XPS measurements of the $\mathrm{Li}(1 \mathrm{~s})$ and $\mathrm{O}(1 \mathrm{~s})$ photoelectron peaks, as a function of exposure time to the residual gases within LTX after lithium deposition on the shell surfaces. The larger uncertainties at shorter exposure times were the result of lower statistics for the XPS data, due to the limited time over which the spectra could be obtained. Within several hours after lithium evaporation, $\mathrm{R}_{\mathrm{Li}, \mathrm{O}}$ reaches a level indicative of a surface that is primarily lithium oxide $\left(\mathrm{Li}_{2} \mathrm{O}\right)$. This is consistent with earlier measurements of the oxidation of lithium when exposed to water vapor.[13]

The $\mathrm{R}_{\mathrm{Li}, \mathrm{O}}$ remains essentially unchanged for nearly 100 hours under a partial pressure of water of about $2 \times 10^{-9}$ torr, and then only slowly begins to decrease. A salient observation is that in terms of LTX plasma performance, a lithium oxide PFC surface does not appear be substantially different from more lithium-dominated surfaces closer to the time lithium was applied. Figure $1 \mathrm{~b}$ shows the maximum discharge current as a function of time after lithium evaporation. High plasma currents are achievable even when the PFCs are primarily lithium oxide, and they continue to be obtained over $\sim 40$ days after lithium evaporation.

Prior to the recent MAPP measurements, it was assumed that enough elemental lithium remained on the PFCs to bind hydrogen when plasma currents at the level shown in Fig. 1b were achieved. This is because the primary mechanism for hydrogen retention was thought to be lithium hydride formation. The new and unexpected result is that good plasma performance can be obtained in the absence of 
elemental lithium on the PFCs, as the MAPP data show. This leads to the conclusion that lithium oxide is apparently able to bind hydrogen.

There is also evidence that the hydrogen binding occurs on a time scale long compared to duration of LTX discharges, but short relative to the period between plasmas. This is illustrated in Fig. 2, which shows the partial pressure of molecular hydrogen, as measured with a residual gas analyzer (RGA), as a function of time. The pressure rises with the introduction of the hydrogen fueling gas before the discharge. During the discharge the hydrogen drops as expected from the fueling of the plasma. The recovery of the pressure to nearly pre-discharge levels suggests the outgassing of the hydrogen retained by the PFCs.

The hydrogen pressure drops by three orders of magnitude by 100 seconds or about three minutes after the termination of the discharge. This longer-term trend is shown on the left side of Fig. 2, where the abscissa is converted to a logarithmic scale. The pressure measurements appear to explain the thermal desorption spectroscopy (TDS) or temperature programmed desorption (TPD) results of the MAPP samples. Heating the samples for TPD significantly alters their surfaces, so XPS was performed first. For good statistics, it took over an hour for XPS data to be obtained. The hydrogen outgassing would have been essentially complete by that time, hence the absence of any hydrogen in the TPD data.

The present experiments thus appear to explain the persistence of low recycling PFCs in LTX. If a lithium oxide layer is formed, there can be sufficient hydrogen retention for low recycling during a discharge. The hydrogen is loosely bound, however, so that it is released well before the next plasma shot occurs. The long-term XPS measurements showed that the partial pressure of water in the LTX vacuum vessel was sufficiently low to limit the formation of lithium hydroxide. Under these conditions, low recycling PFC surfaces could be maintained long after lithium was applied to their surfaces.

The ability of lithium oxide to retain hydrogenic species is supported by laboratory studies of thin lithium films on a molybdenum (TZM) substrate.[14] In the absence of oxygen, deuterium was retained in the form of lithium deuteride after exposure to a flux of diatomic deuterium ions at room temperature. This was indicated by the appearance of a peak in the TPD spectrum that corresponded to the decomposition of lithium deuteride (Fig. 3). If the lithium was allowed to oxidize and subsequently exposed to the same deuterium flux, a peak appears in the TPD spectrum that is indicative of deuterium retention. The spectra in Fig. 3 show the start of deuterium release at approximately $400 \mathrm{~K}$. The deuterium desorption could still extrapolate to a small but finite rate, however, at about $300 \mathrm{~K}$. It might then be possible for the LTX PFCs to outgas at room temperature on time scales consistent with the RGA and TPD data.

The data in Fig. 3 also show that there was no longer a peak corresponding to the decomposition of lithium deuteride. This could be analogous to what was found with graphite PFCs, where the binding of the lithium in a complex with carbon and oxygen inhibits the formation of lithium deuteride. However, the lithium-carbon-oxygen complex is still able to bind deuterium. Similarly, the lithium oxide in the film on the TZM substrate inhibits the formation of lithium deuteride. Instead of retention in this compound, the LTX and laboratory results would then be consistent with oxygen (in the form of lithium oxide) having a major role in binding hydrogenic species in PFC surfaces.

\section{Conclusions and Implications for Lithium Applied to High-Z Plasma-Facing Components}

Experiments in LTX and surface science laboratories at the Princeton Plasma Physics Laboratory and the University of Illinois at Urbana-Champaign have shown that complexes involving lithium and oxygen are capable of retaining hydrogenic species in high- $Z$ as well as graphite PFCs. This is supported by measurements performed with the Liquid Lithium Divertor (LLD) in NSTX. The LLD as it was located in the lower divertor is shown in Fig. 4. The LLD surface consisted of $\sim 150 \square$ m-thick porous molybdenum that was plasma-sprayed on top of a $250 \mathrm{~mm}$-thick stainless steel substrate. 
Lithium was then applied to the surface from evaporators located in the upper dome of NSTX.[15] It is thus likely that like the LTX shell surfaces, the LLD became a high-Z PFC with a lithium oxide coating.

Fast visible cameras had views of the lower NSTX divertor.[16] Fig. 5 shows visible camera images of this region through two different filters. The numbers correspond to the LLD segments in Fig. 4, which are mapped to be linear in the images. The upper image records the deuterium-alpha emission. The bright band appears curved, because it corresponds to the plasma strike point on the vertical section of the inboard divertor in Fig. 4. The lower image records the neutral lithium emission.

Each of the four toroidal LLD segments was installed with a set of heaters that could be controlled independently. Only three were operational, and they were used to heat segments 1,2 , and 4 to $220^{\circ} \mathrm{C}$. The higher deuterium alpha emission for the three heated segments suggests the release of deuterium. The brighter neutral lithium emission is consistent with the heating of the segments, as higher sputtering is expected with increasing temperature.

The results from heating the LLD were similar to laboratory observations of deuterium retention in thin lithium films. Both experiments involved lithium oxide on a molybdenum substrate, and the deuterium alpha emission from the LLD occurred at about the same temperature the deuterium peak appeared in the TPD spectrum for the oxidized lithium film.

These results have implications for lithium conditioning when the NSTX-U PFCs are upgraded. The existing graphite tiles are to be replaced with TZM tiles, but the primary means for applying lithium to their surfaces will remain lithium evaporation. If deuterium retention can occur with lithium oxide and does not require the formation of lithium deuteride, it may relax the need to maximize the amount of elemental lithium on most PFC surfaces in the near term to reduce recycling.

The major long-term challenge is to extend lithium coverage into the divertor strike point region. This will require a scheme to flow liquid lithium, and different options are being explored.
As an intermediate step prior to the implementation of a fully-flowing liquid lithium divertor, tiles that are prefilled with candidate liquid metals are under consideration for high- $Z$ PFCs in NSTX-U.[17] The liquid metal would be transported from the interior of the tile by a wick in contact with a prefilled reservoir. Like the Capillary Porous System (CPS)[18], the PFC surface would be textured to enable wetting.

Note that the digital data for Fig. 1 this paper can be found at http://arks.princeton.edu/ark:/88435/dsp01x920 g025r.

\section{Acknowledgements:}

This work was supported by US Department of Energy Contracts DE-AC02-09CH11466, DE-SC0010717, and DE-AC52-07NA27344.

[1] D. K. Mansfield et al., Phys. Plasmas 3, 1892 (1996)

[2] M. G. Bell et al., Plasma Phys. Control. Fusion 51, 124054 (2009)

[3] R. Majeski et al., Phys. Rev. Letters 97, 075002 (2006)

[4] J. C. Schmitt et al., Phys. Plasmas 22, 056112 (2015)

[5] C. S. Liu, M. N. Rosenbluth, and C. W. Horton, Jr. Phys. Rev. Lett. 29, 1489 (1972)

[6] J.D. Strachan et al., Plasma Phys. Controlled Fusion 36, B3 (1994)

[7] D. Ernst et al. Phys. Rev. Lett. 81, 2454 (1998)Tobita K. et al 2009 Nucl. Fusion 49 075029

[8] M.J. Baldwin, R.P. Doerner, S.C. Luckhardt, and R.W. Conn, Nucl. Fusion 42, 1318 (2002)

[9] C. N. Taylor et al., Fus. Eng. Design 88, 3157 (2013)

[10] P. Krstic et al., Phys. Rev. Letters 110, 105001 (2013)

[11] C. N. Taylor et al., Rev. Sci. Instruments 83, 10D703 (2012)

[12] M. Lucia et al., J. Nucl. Materials 463, 907 (2015)

[13] C. H. Skinner et al., J. Nucl. Materials 438, 5647 (2013) 
[14] A. Capece et al., J. Nucl. Materials 463, 1177 (2015)

[15] H. Kugel et al., Fus. Eng. Design 87, 1724 $\underline{(2012)}$

[16] F. Scotti et al., Rev. Sci. Instruments 83, 10E532 (2012)

[17] M. Jaworski et al., "Incremental upgrades toward high-heat flux, liquid lithium PFCs in the NSTX-U," submitted for publication in Fus. Eng. Design

[18] S. Mirnov et al., Fusion Eng. Design 65, 455 (2003) 

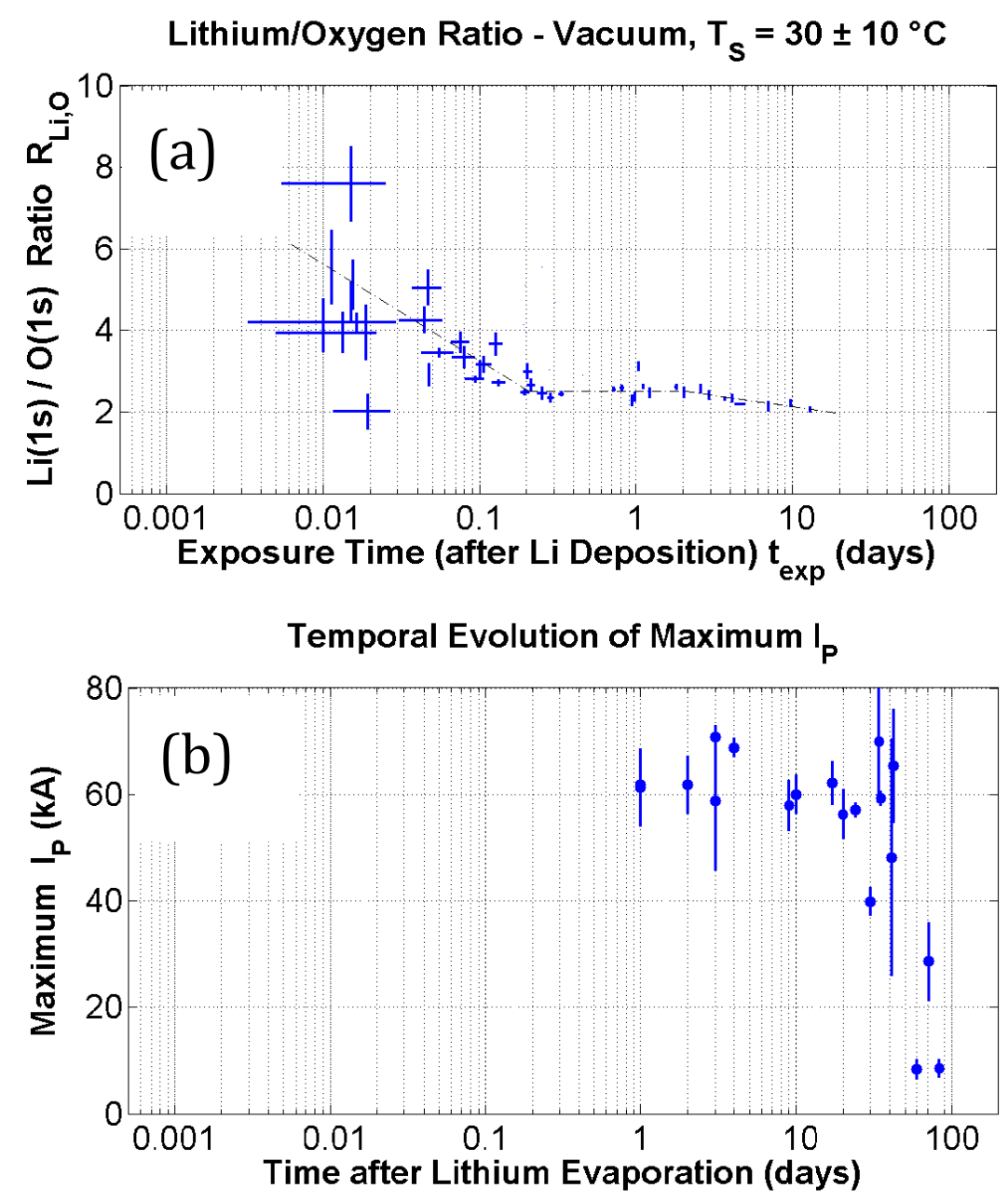

Fig. 1. (a) Lithium to oxygen ratios obtained with XPS as a function of exposure time after lithium deposition on LTX shell surfaces. (b) Maximum plasma current achieved in LTX as a function of time after lithium deposition on LTX shell surfaces. Note that the units in the abscissa on each plot are in days. 


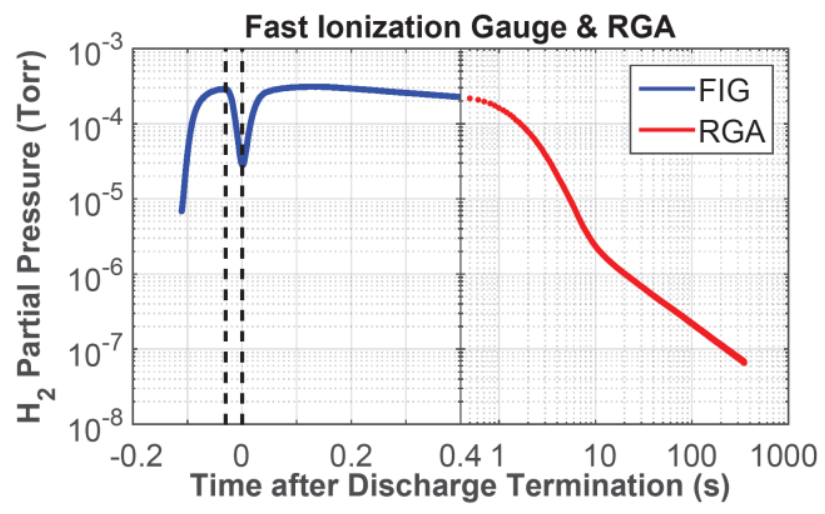

Fig. 2. Residual gas analyzer (RGA) measurements of the partial pressure of molecular hydrogen from the introduction of the fueling gas shortly before the plasma shot until several hundred seconds after the termination of the discharge. Note that the abscissa changes from a linear to a logarithmic time scale at $0.4 \mathrm{~s}$. 


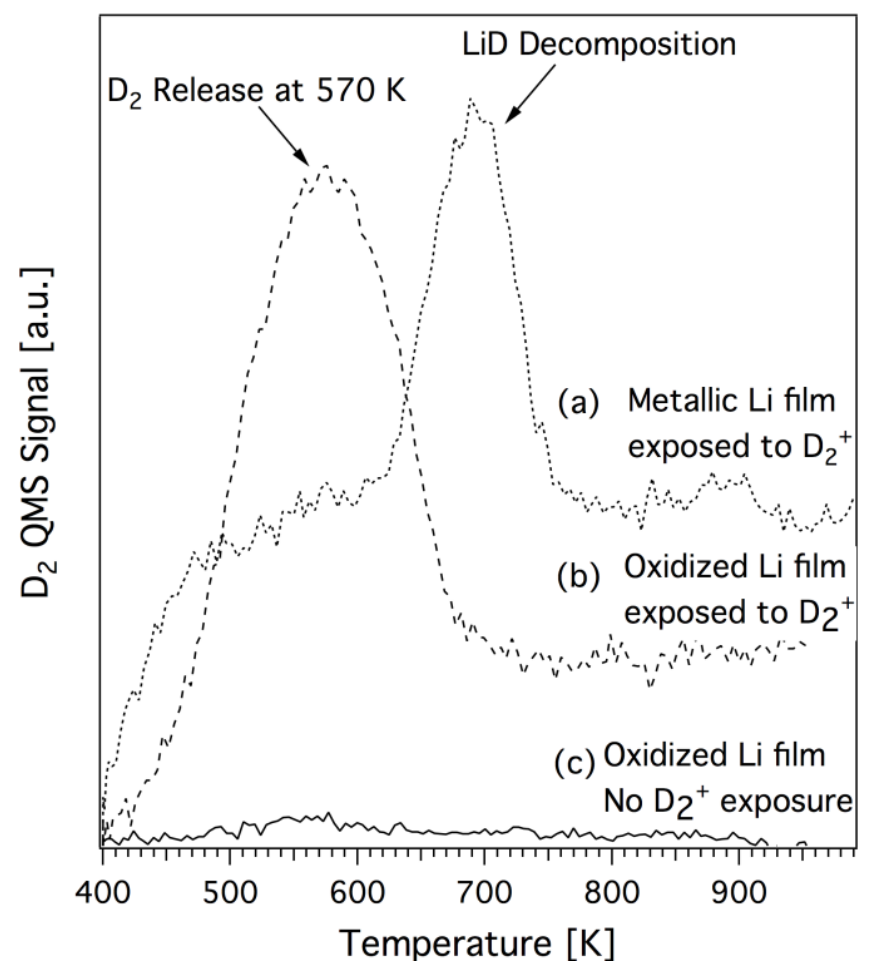

Fig. 3 - Molecular deuterium temperature programmed desorption (TPD) from a 3 monolayer lithium film exposed to $265 \mathrm{~V}$ singly-ionized molecular deuterium at a fluence of $2 \times 10^{15}$ $\mathrm{D} / \mathrm{cm}^{2}$ at $400 \mathrm{~K}$. The three cases shown correspond to (a) a metallic lithium film exposed to deuterium, (b) an oxidized lithium film exposed to deuterium, and (c) an oxidized lithium film that was not exposed to deuterium. Reprinted with permission from A. Capece et al., J. Nucl. Materials 463, 1177 (2015). 


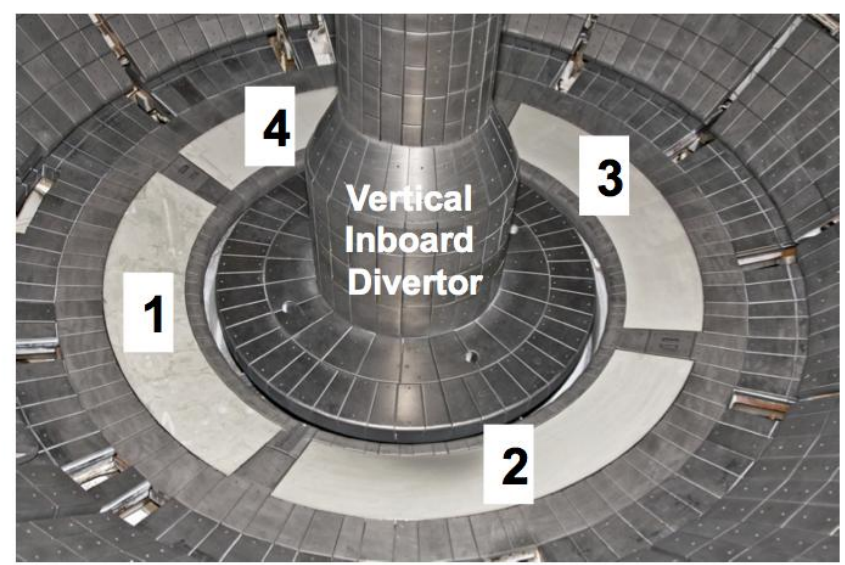

Fig. 4 - Interior of NSTX showing LLD segments in lower divertor region. 


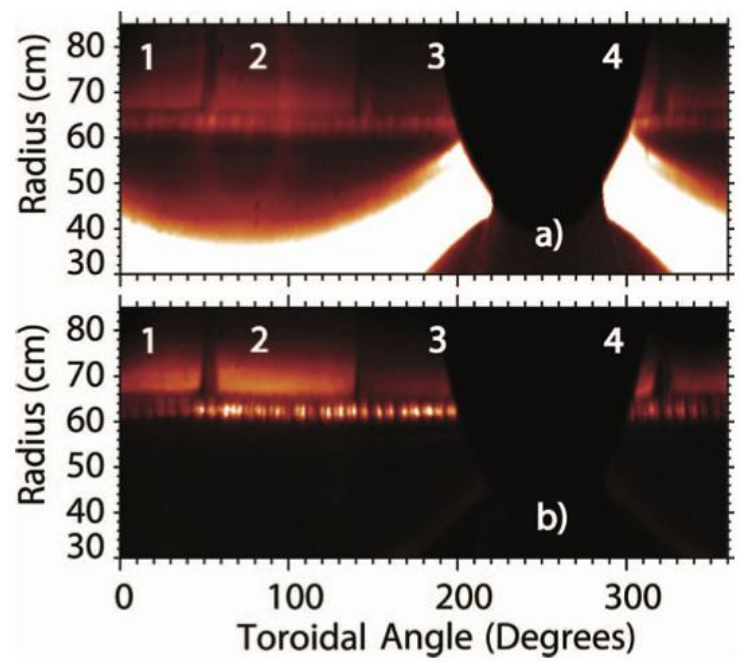

Fig. 5 - Filtered visible camera images showing (a) deuterium alpha ( $\mathrm{D}_{\square}$ ) emission and (b) atomic lithium (LiI) emission from lower NSTX divertor during plasma shot. Reprinted with permission from F. Scotti et al., Rev. Sci. Instruments 83, 10E532 (2012). 